\title{
Two-State Wave Packet for Strong Field-Free Molecular Orientation
}

\author{
Sebastian Trippel, ${ }^{1}$ Terry Mullins, ${ }^{1}$ Nele L. M. Müller, ${ }^{1}$ Jens S. Kienitz, ${ }^{1,2}$ \\ Rosario González-Férez, ${ }^{2,3}$ and Jochen Küpper ${ }^{1,2,4, *}$ \\ ${ }^{1}$ Center for Free-Electron Laser Science, DESY, Notkestrasse 85, 22607 Hamburg, Germany \\ ${ }^{2}$ The Hamburg Center for Ultrafast Imaging, University of Hamburg, Luruper Chaussee 149, 22761 Hamburg, Germany \\ ${ }^{3}$ Instituto Carlos I de Física Teórica y Computacional and Departamento de Física Atómica, Molecular y Nuclear, \\ Universidad de Granada, 18071 Granada, Spain \\ ${ }^{4}$ Department of Physics, University of Hamburg, Luruper Chaussee 149, 22761 Hamburg, Germany
}

(Received 9 September 2014; published 10 March 2015)

\begin{abstract}
We demonstrate strong laser-field-free orientation of absolute-ground-state carbonyl sulfide molecules. The molecules are oriented by the combination of a 485-ps-long nonresonant laser pulse and a weak static electric field. The edges of the laser pulse create a coherent superposition of two rotational states resulting in revivals of strong transient molecular orientation after the laser pulse. The experimentally attained degree of orientation $\langle\cos \theta\rangle \approx 0.6$ corresponds to the theoretical maximum for mixing of the two states. Switching off the dc field would provide the same orientation completely field free.
\end{abstract}

DOI: 10.1103/PhysRevLett.114.103003

PACS numbers: 37.10.-x, 37.20.+j, 82.20.Bc

Fixed-in-space samples of molecules have attracted wide interest for diverse applications. These include stereochemistry [1-3] as well as molecular imaging using photoelectron angular distributions [4-8], high-order harmonic generation [9-11], or electron and x-ray diffraction [12,13]. Traditionally, state selection [14] and brute-force orientation [2] have been used to create oriented samples [15]. Stronger, also three-dimensional, orientation has been achieved through the use of a combination of electrostatic and strong nonresonant laser fields [16-24]. However, in these approaches the presence of a strong electrostatic or laser field may influence the outcome of the experiments. Therefore, it is of particular interest to create oriented molecules in essentially field-free space.

Laser-field-free orientation has been achieved by the combination of strong static electric fields and shaped laser pulses $[21,25]$ and through two-color femtosecond laser pulses $[26,27]$. The latter yields oriented molecules in the absence of any external field at the rotational revivals of the molecule. However, the achievable degree of orientation is weak, limited by the onset of ionization [27,28]. Extending this method by an additional, correctly timed, strong alignment prepulse allows for significant degrees of orientation without much ionization for small molecules [11,29]. Singlecycle terahertz pulses provide an alternative approach to field-free orientation via direct resonant rotational excitation $[30,31]$. The experimentally realized degree of orientation with a single terahertz pulse is small, due to difficulties in matching the radiation spectrum to the excitation resonances, especially for the small energy gaps between low-energy rotational states of small molecules. Again, improved orientation is obtained by applying an appropriately timed nonresonant alignment prepulse to create coherent superpositions with contributions in higher-energy states $[32,33]$.

Here, we report on the creation of a laser-field-free strongly oriented molecular sample from absolute-ground- state-selected carbonyl sulfide (OCS) molecules using mixed-field orientation. Our experimental approach is novel, unique, and generally applicable to a wide number of molecules, also in excited rotational states. It is easily implemented using standard commercial laser systems.

Conceptionally, we exploit nonadiabatic couplings between pendular states in mixed-field orientation to mimic the effects of the terahertz-pulse-orientation approach. Even for small molecules these couplings are highly efficient, which results in a much stronger orientation compared to short terahertz pulses. Our method relies on the molecule's static polarizability and its dipole moment. It is complementary to two-color experiments based on the interaction with the molecule's hyperpolarizability $[11,26]$. Thus, the approaches are useful for different scenarios, i.e., different molecules.

While in the case of laser alignment an adiabatic response of the system to the laser field is provided if all time scales of the laser pulse are longer than the rotational period of the molecule [34,35], this is not the case for orientation in combined laser and static electric fields $[23,36]$. Even for a rapidly rotating molecule, such as OCS with a rotational period of $\tau_{\text {rot }} \approx 82 \mathrm{ps,} \mathrm{a} \mathrm{laser} \mathrm{pulse}$ duration of $\sim 50 \mathrm{~ns}$ would be required to adiabatically orient the molecules in a moderate static electric field of $1 \mathrm{kV} / \mathrm{cm}$ [37]. This is due to the coupling between the oriented $|\tilde{0}, \tilde{0}\rangle$ and antioriented $|\tilde{1}, \tilde{0}\rangle$ states [38].

We demonstrate how this $\Delta J=1$ coupling can be utilized, similar to the coupling by resonant terahertz fields, to create a coherent wave packet between the $J=0$ and $J=1$ rotational states and to obtain orientation revivals after the laser pulse. We show that we can strongly control the mixing of the states by simply adjusting the laser power. The rise and fall times of the laser pulses have been chosen to be long enough to avoid couplings with $\Delta J>1$, but short enough to be strongly nonadiabatic for $\Delta J=1$ 
couplings. This results in a coherent superposition of rotational states with very-strong-orientation revivals after the laser pulse is switched off.

A schematic of the experimental setup is shown in Fig. 1. A pulsed molecular beam was provided by expanding $500 \mathrm{ppm}$ of OCS seeded in 6 bar of neon through a cantilever piezo valve [39] at a repetition rate of $250 \mathrm{~Hz}$. The molecules were dispersed according to their quantum state by the electric deflector [40], and a pure sample of ground-state OCS was selected [41]. These molecules were oriented by the combined action of a moderately intense, 485-ps-long laser pulse $\left(I_{\text {control }} \approx 10^{11} \mathrm{~W} / \mathrm{cm}^{2}\right)$ and a weak dc electric field $\left(E_{\text {stat }}=840 \mathrm{~V} / \mathrm{cm}\right)$ inside a velocity map imaging (VMI) spectrometer. The rise and fall times of the laser pulse are $130 \mathrm{ps}$. The polarization of the control laser had an angle $\beta=45^{\circ}$ with respect to the static electric field. The angular confinement was probed through strong-field multiple ionization by a linearly polarized, $30 \mathrm{fs}$ laser pulse $\left(I_{\text {probe }}=3 \times 10^{14} \mathrm{~W} / \mathrm{cm}^{2}\right)$, resulting in Coulomb explosion of the molecule. The polarization of the probe laser was always perpendicular to the polarization of the orientation laser. The produced ions were velocity mapped onto a position sensitive detector. The detected $\mathrm{S}^{+}$ion distribution from the Coulomb fragmentation channel $\mathrm{OCS}+n h \nu \rightarrow \mathrm{OC}^{+}+\mathrm{S}^{+}$was highly directional and provided direct information on the alignment and orientation of the OCS molecules at the time of ionization.

The control and probe laser pulses were provided by an amplified femtosecond laser system [42]. The probe pulses had pulse energies of $200 \mu \mathrm{J}$ and a beam waist of $\omega_{0}=36 \mu \mathrm{m}$. The control pulses had energies controlled between 0 and $7 \mathrm{~mJ}$ and a beam waist of $\omega_{0}=70 \mu \mathrm{m}$. Since both beams were generated by the same laser system they were inherently synchronized. The relative timing between the two pulses was adjusted by a motorized linear translation stage.

To obtain insight into the angular dynamics the degree of orientation [43] was recorded for a range of laser peak intensities $I_{\text {control }}$ as a function of time. For each time delay and intensity of the laser pulse, a projection of the threedimensional $\mathrm{S}^{+}$velocity distribution onto the twodimensional detector was recorded. The two-dimensional

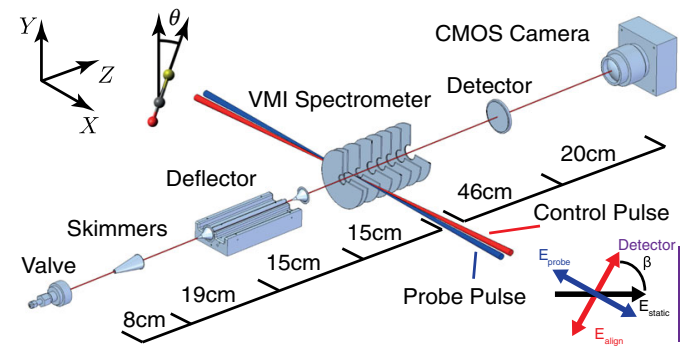

FIG. 1 (color online). Schematic of the experimental setup, including the axis system and the definition of angles $\theta$ between the laboratory-fixed $Y$ axis and the molecule-fixed $z$ axis. The angle $\beta$ defines the angle between the polarization axis of the orientation laser and the static electric field of the VMI spectrometer. velocity distributions showed a rich structure due to different fragmentation channels of OCS after Coulomb explosion. In order to resolve the different channels a mixing angle of $\beta=45^{\circ}$ has been chosen [40].

The degrees of alignment and orientation [43] were determined from the distribution of velocity components parallel to the detector surface $\left(v_{\|}\right)$with $2800<v_{\|}<$ $5400 \mathrm{~m} / \mathrm{s}$. This corresponds to $\mathrm{S}^{+}$ions from the $\mathrm{OC}^{+}+$ $\mathrm{S}^{+}$channel (vide supra). The contribution from other fragmentation channels is estimated to be below $10 \%$ [44]. A 2D representation of the experimental results is shown in Fig. 2(a) for $\beta=+45^{\circ}$. We focus on the postpulse orientation dynamics following the falling edge of the control laser pulse at $\sim 750 \mathrm{ps}$.

During the laser pulse OCS was oriented due to mixedfield orientation. After the laser pulse a strong oscillatory behavior was observed. These oscillations correspond to the wave packet dynamics of a coherent superposition of the $|0,0\rangle$ and $|1,0\rangle$ states. With increasing laser intensity $I_{\text {control }}$ the degree of orientation increased and the oscillation maxima shifted to longer delays.

Figure 2(b) shows the degree of alignment obtained from the same data as Fig. 2(a) $\left(\beta=+45^{\circ}\right)$. Even without an alignment pulse $\left(I_{\text {control }}=0\right)$ some permanent alignment was present, $\left\langle\cos ^{2} \theta_{2 \mathrm{D}}\right\rangle>0.5$, which increased with $I_{\text {control }}$. This might, partly, be due to so-called geometric alignment, i.e., selective ionization of OCS by the perpendicularly polarized probe laser and, mostly, be due to permanent alignment through the population of the $|1,0\rangle$ state. Classically speaking, the molecules rotated in planes containing the control laser polarization vector. However, no revival structures were

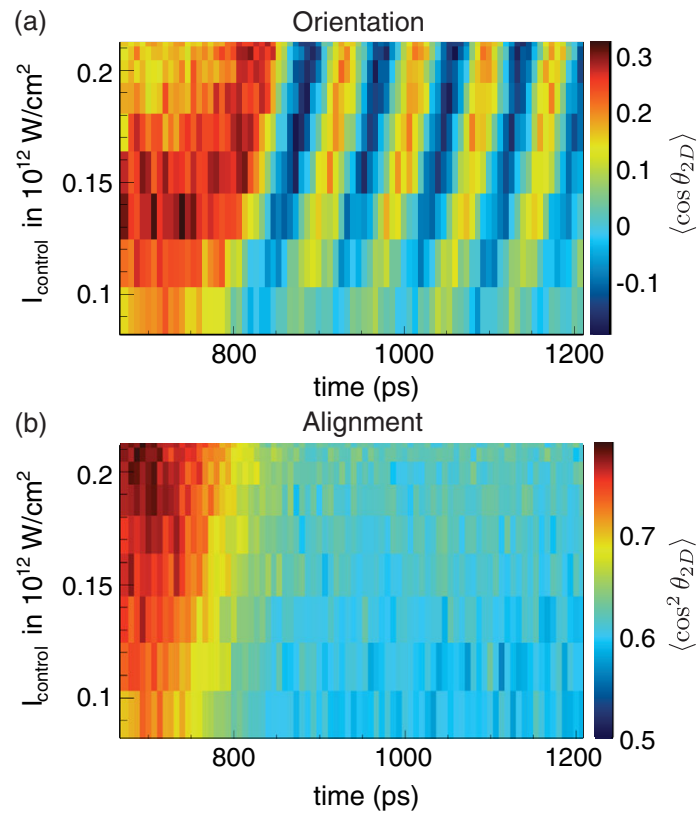

FIG. 2 (color online). Degree of orientation $\left\langle\cos \theta_{2 \mathrm{D}}\right\rangle$ [43] of OCS with (a) $\beta=+45^{\circ}$ as a function of the relative delay between the orientation and probe laser pulses and the control laser peak intensity. (b) Degree of alignment $\left\langle\cos ^{2} \theta_{2 \mathrm{D}}\right\rangle$ [43] extracted from the same data set as (a). 
observed, demonstrating the (quasi)adiabatic alignment dynamics under these conditions [35,42].

Calculations that include the experimental temporal control laser intensity profile show that the rotational dynamics is dominated by the coupling of two states, $|0,0\rangle$ and $|1,0\rangle$ [37]. In this two-state model the nonadiabatic coupling between the field-dressed $|\tilde{0}, \tilde{0}\rangle$ and $|\tilde{1}, \tilde{0}\rangle$ states creates a wave packet of the rotational states $|0,0\rangle$ and $|1,0\rangle$, which results in field-free orientation and antiorientation. As soon as the laser pulse is switched off this results in a time dependent wave packet of the form

$|\Psi(t)\rangle=\left|c_{00}\right||0,0\rangle e^{-i\left(E_{00} t / \hbar-\phi_{00}\right)}+\left|c_{10}\right||1,0\rangle e^{-i\left(E_{10} t / \hbar-\phi_{10}\right)}$,

where $E_{l m}$ and $\phi_{l m}$ denote the energies and the phases of the states $|l, m\rangle$, which are spherical harmonics $Y_{l}^{m}$. The time dependent degree of orientation results in

$$
\begin{aligned}
\langle\cos \theta\rangle(t)= & 2\left|c_{00}\right| \sqrt{1-\left|c_{00}\right|^{2}} \\
& \times\langle 0,0|\cos \theta| 1,0\rangle \cos (\Delta E t / \hbar+\Delta \phi)
\end{aligned}
$$

with $\Delta E=E_{10}-E_{00}, \Delta \phi=\phi_{00}-\phi_{10}$, and $\left|c_{00}\right|^{2}+\left|c_{10}\right|^{2}=1$. The maximum degree of orientation is given by $\sqrt{1 / 3} \approx 0.577$, which is obtained at $\left|c_{00}\right|=\sqrt{1 / 2}$. A similar consideration for the degree of alignment results in

$$
\left\langle\cos ^{2} \theta\right\rangle=\frac{3}{5}-\frac{4}{15}\left|c_{00}\right|^{2} .
$$

Thus, while the degree of orientation shows a time dependent variation, the degree of alignment is time independent. The latter does, however, show a dependence on the weight of the ground state after the orientation pulse in agreement with the experiment.

Figure 3(a) shows the temporal experimental evolution of the degree of orientation during and after the laser pulse at a peak intensity of $0.215 \mathrm{TW} / \mathrm{cm}^{2}$. The control laser pulse is indicated by the light gray area. Before the laser pulse was present we observed a small orientation of the molecular sample $\left(\left\langle\cos \theta_{2 \mathrm{D}}\right\rangle \approx 0.01\right)$. This is due to "bruteforce" orientation via the static electric field of the VMI spectrometer [22]. The calculated weights of the $|\tilde{0}, \tilde{0}\rangle$, $|\tilde{1}, \tilde{0}\rangle$, and $|\tilde{1}, \tilde{1}\rangle$ states are $\left|c_{00}\right|^{2}=0.99980,\left|c_{10}\right|^{2}=$ $1.5 \times 10^{-4}$, and $\left|c_{11}\right|^{2}=5 \times 10^{-5}$, respectively. All other states have weights below $1.4 \times 10^{-9}$. As soon as the laser pulse began the orientation increased, and it continued to do so over the entire laser pulse. This dynamics of the orientation is the beginning of an oscillating wave packet of the two pendular states $|\tilde{0}, \tilde{0}\rangle$ and $|\tilde{1}, \tilde{0}\rangle$ formed by the fast rise at the beginning of the laser pulse. It is equivalent to the field-free wave packet described above, but with a much longer period due to the near degeneracy of the pendular states in the strong laser field. The maximum orientation during the laser pulse would only be reached after about
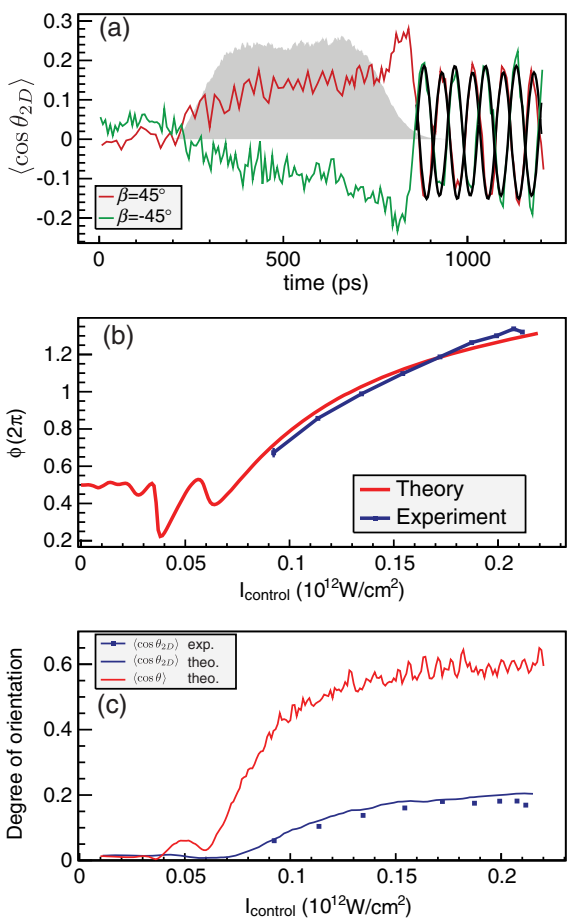

FIG. 3 (color online). (a) Experimental temporal evolution of the degree of orientation and at a peak intensity of $0.215 \mathrm{TW} / \mathrm{cm}^{2}$. Black lines result from a fit of Eq. (4) to the data points. (b) Phase shift of the postpulse dynamics as a function of the peak intensity of the control laser pulse, in units of $2 \pi$. (c) Degree of orientation as a function of the peak intensity of the control laser pulse. Statistical errors are smaller than the size of the markers.

$1 \mathrm{~ns}$ for the weakest control-laser fields, or later for higher laser intensities. Therefore, we do not reach this maximum during our 485-ps-long laser pulses. A strong enhancement was observed at the end of the laser pulse. This is due to both the strong mixing of the states due to nonresonant couplings provided by the falling edge of the laser pulse and the change in the beating frequency due to the decrease of the laser intensity. As soon as the laser pulse was switched off the field-free oscillatory behavior was observed (vide supra). The phase of the postpulse dynamics depends on the phase of the beating during the laser pulse. Thus, it depends on both the control-laser intensity and its pulse duration.

We fitted the following function to the postpulse dynamics:

$$
f(t)=a+b \cos \left(\frac{2 \pi t}{T}+\phi\right)
$$

with $b>0$. The fits results are shown as black lines in Fig. 3(a). The period $T$ of the oscillation was determined to be $83 \mathrm{ps}$. This is the $1 /(2 B c)$ revival period expected from the coupling of the states $|0,0\rangle$ and $|1,0\rangle$. The phase of the oscillation was shifted by $\pi$ when the laser polarization was rotated by $90^{\circ}$ (green line). In addition, a small vertical offset $a$, due to a small contribution of the $|2,0\rangle$ 
state, was observed. While this does not significantly change the cosine form of the postpulse dynamics (see the Supplemental Material [45]), the correspondingly increased peak orientation to one side of the laser polarization axis might have implications on the results of experiments when the outcome is strongly dependent on the degree of molecular orientation. The postpulse timeaveraged degree of orientation is still zero.

Figure 3(b) shows the phase $\phi$ of the postpulse dynamics obtained from the cosine fit (4) as a function of the peak intensity of the control laser pulse (blue line). After a minimization of the temporal offset between theory and experiment by a least squares fit the theoretically calculated phase between the weights $c_{00}$ and $c_{10}$ was observed (red line). This phase shift as a function of $I_{\text {control }}$ is an observable of the postpulse dynamics that is fully independent of the influence of the probe pulse. The excellent agreement shows that the measured orientation dynamics at the peak intensity of the orientation pulse is very well described by our theoretical model.

Figure 3(c) shows the maximum in the degree of orientation in the postpulse dynamics, $a+b$ in Eq. (4), as a function of $I_{\text {control }}$. Experimental values are shown as blue dots and theoretical values, including the probe-laser selectivity and the volume effect [36], are shown as a blue line. The corresponding three-dimensional degree of orientation obtained from the same calculations is shown as a red line. It increases with $I_{\text {control }}$ until it saturates at $\langle\cos \theta\rangle=0.6$. This shows that at high intensities the mixing of the two states $|0,0\rangle$ and $|1,0\rangle$ is practically optimal for the degree of orientation. The influence of the multiply ionizing probe laser strongly changes the observation of the degree of orientation. Therefore, the observed orientation is lower than the real orientation of the molecular sample. The high frequency oscillation in the degree of orientation can be attributed to the influence of the state $|2,0\rangle$, which has a different phase for every intensity. The slightly lower measured 2D degree of orientation, compared to our calculations, can be attributed to small differences regarding the modeling of the probe pulse distribution, the volume effect, and small contributions from other fragmentation channels.

In conclusion, 485-ps-long moderately strong $\left(10^{11} \mathrm{~W} / \mathrm{cm}^{2}\right)$ laser pulses were used to induce laserfield-free transient orientation in quantum-state-selected ground-state OCS molecules. In combination with our simulations we infer that strong orientation with $\langle\cos \theta\rangle=$ 0.6 was achieved through coherent coupling of the fieldfree $|0,0\rangle$ and $|1,0\rangle$ rotational states. This corresponds to 93\% of the molecules pointing in the same direction along the $I_{\text {control }}$ laser polarization axis. Since only two states are involved in the wave packet, the orientation dynamics is slow and strong field-free orientation can be obtained for a duration of several picoseconds. This is long enough to study most molecular dynamics processes in moderately sized molecules during that period [46].
The demonstrated degree of field-free orientation is 6 times larger than that previously observed via coherent rotational excitation in combination with terahertz pulses [33]. It is also a clear improvement over the $73 \%-83 \%$ directionality achieved in a complex two-pulse two-color experiment [11]. Moreover, the latter experiments relied on very strong laser fields of $5 \times 10^{13} \mathrm{~W} / \mathrm{cm}^{2}$. These intensities would simply destroy most molecules through ionization, and lowering the intensity would sharply decrease the achievable orientation due to the cubic scaling of the hyperpolarizability interaction. To the contrary, our approach is generally applicable for heteronuclear molecules. This includes large molecules with their reduced ionization thresholds.

In contrast to single-cycle terahertz pulses, the nonresonant interactions in our scheme couple energetically neighboring states very efficiently, independent of their rotational excitation. This opens up the possibility to create even more strongly field-free-oriented samples by preparing the molecules in high $J$ states before the mixed-field orientation pulse $[11,21,33,47]$. However, the current samples with molecules in a very small number of quantum states are advantageous for state-specific experiments such as state-to-state reaction stereodynamics. Simulations show that rapidly turning off the VMI field, down to $\tau=100 \mathrm{ps}$, would not alter the wave packet dynamics and result in strong fully field-free orientation. Furthermore, the maximum orientation of the postpulse dynamics can be controlled, i.e., enhanced or suppressed, by the exact timing of the switching off of the pulse. In our field configuration, to efficiently transfer the population back to the ground state [35], the falling edge of the laser pulse should start after a few nanoseconds, due to the slow field-dressed dynamics of the near-degenerate pendular states in the strong laser field.

Our approach holds in general for all states of a linear molecule, not only for the absolute ground state. We show that the mixing of the states can be controlled between zero and complete mixing, with a weight of $1 / \sqrt{2}$ for both states, by simply adjusting the laser power. Our simulations show that only the rise and fall times of the laser pulse and its intensity are relevant for the control of the orientation, at typical dc electric field strengths in VMI spectrometers and for a given angle $\beta$. The same orientation could be achieved using a transform limited laser pulse with a pulse duration of $130 \mathrm{ps}$ and a pulse energy of $2 \mathrm{~mJ}$ focused to $\omega_{0}=50 \mu \mathrm{m}$. Such laser parameters are easily accessible by stretching commercial femtosecond lasers or directly using the output of a chirped-pulse amplifier.

We gratefully acknowledge helpful discussions with Henrik Stapelfeldt. In addition to DESY, this work has been supported by the excellence cluster "The Hamburg Center for Ultrafast Imaging - Structure, Dynamics and Control of Matter at the Atomic Scale" of the Deutsche Forschungsgemeinschaft, including the Mildred Dresselhaus Award for R. G. F., R. G. F. also gratefully acknowledges financial support by the Spanish project FIS2011-24540 (MICINN), 
Grants No. P11-FQM-7276 and No. FQM-4643 (Junta de Andalucía), and by the Andalusian research group FQM207. N. L. M. M. gratefully acknowledges a fellowship of the Joachim Herz Stiftung.

*jochen.kuepper@cfel.de; http://desy.cfel.de/cid/cmi

[1] P. R. Brooks, Science 193, 11 (1976).

[2] H. J. Loesch and A. Remscheid, J. Chem. Phys. 93, 4779 (1990).

[3] T. P. Rakitzis, A. J. van den Brom, and M. H. M. Janssen, Science 303, 1852 (2004).

[4] M. Meckel, D. Comtois, D. Zeidler, A. Staudte, D. Pavicic, H. C. Bandulet, H. Pepin, J. C. Kieffer, R. Doerner, D. M. Villeneuve, and P. B. Corkum, Science 320, 1478 (2008).

[5] C. Z. Bisgaard, O. J. Clarkin, G. Wu, A. M. D. Lee, O. Geßner, C. C. Hayden, and A. Stolow, Science 323, 1464 (2009).

[6] L. Holmegaard, J. L. Hansen, L. Kalhøj, S. L. Kragh, H. Stapelfeldt, F. Filsinger, J. Küpper, G. Meijer, D. Dimitrovski, M. Abu-samha, C. P. J. Martiny, and L. B. Madsen, Nat. Phys. 6, 428 (2010).

[7] F. Kelkensberg, A. Rouzée, W. Siu, G. Gademann, P. Johnsson, M. Lucchini, R. R. Lucchese, and M. J. J. Vrakking, Phys. Rev. A 84, 051404 (2011).

[8] R. Boll et al., Phys. Rev. A 88, 061402(R) (2013).

[9] J. Itatani, J. Levesque, D. Zeidler, H. Niikura, H. Pépin, J. C. Kieffer, P. B. Corkum, and D. M. Villeneuve, Nature (London) 432, 867 (2004).

[10] C. Vozzi, M. Negro, F. Calegari, G. Sansone, M. Nisoli, S. De Silvestri, and S. Stagira, Nat. Phys. 7, 822 (2011).

[11] P. M. Kraus, D. Baykusheva, and H. J. Wörner, Phys. Rev. Lett. 113, 023001 (2014); arXiv:1311.3923.

[12] C. J. Hensley, J. Yang, and M. Centurion, Phys. Rev. Lett. 109, 133202 (2012)

[13] J. Küpper et al., Phys. Rev. Lett. 112, 083002 (2014); arXiv:1307.4577.

[14] J. Reuss, in Atomic and Molecular Beam Methods, edited by G. Scoles (Oxford University Press, New York, 1988), Vol. 1, Chap. 11, pp. 276-292.

[15] Alignment refers to the angular confinement of moleculefixed axes along laboratory-fixed axes. Orientation, in addition, refers to the dipole moments of the molecules pointing in a particular direction in space.

[16] B. Friedrich and D. Herschbach, J. Chem. Phys. 111, 6157 (1999).

[17] R. Baumfalk, N. H. Nahler, and U. Buck, J. Chem. Phys. 114, 4755 (2001).

[18] H. Sakai, S. Minemoto, H. Nanjo, H. Tanji, and T. Suzuki, Phys. Rev. Lett. 90, 083001 (2003).

[19] H. Tanji, S. Minemoto, and H. Sakai, Phys. Rev. A 72, 063401 (2005).

[20] L. Holmegaard, J. H. Nielsen, I. Nevo, H. Stapelfeldt, F. Filsinger, J. Küpper, and G. Meijer, Phys. Rev. Lett. 102, 023001 (2009); arXiv:0810.2307.

[21] O. Ghafur, A. Rouzee, A. Gijsbertsen, W. K. Siu, S. Stolte, and M. J. J. Vrakking, Nat. Phys. 5, 289 (2009).

[22] I. Nevo, L. Holmegaard, J. H. Nielsen, J. L. Hansen, H. Stapelfeldt, F. Filsinger, G. Meijer, and J. Küpper, Phys. Chem. Chem. Phys. 11, 9912 (2009).

[23] J. H. Nielsen, H. Stapelfeldt, J. Küpper, B. Friedrich, J. J. Omiste, and R. González-Férez, Phys. Rev. Lett. 108, 193001 (2012); arXiv:1204.2685.
[24] J. L. Hansen, J. J. Omiste Romero, J. H. Nielsen, D. Pentlehner, J. Küpper, R. González-Férez, and H. Stapelfeldt, J. Chem. Phys. 139, 234313 (2013); arXiv:1308.1216.

[25] A. Goban, S. Minemoto, and H. Sakai, Phys. Rev. Lett. 101, 013001 (2008).

[26] S. De, I. Znakovskaya, D. Ray, F. Anis, N. G. Johnson, I. A. Bocharova, M. Magrakvelidze, B. D. Esry, C. L. Cocke, I. V. Litvinyuk, and M. F. Kling, Phys. Rev. Lett. 103, 153002 (2009); arXiv:0907.3250.

[27] I. Znakovskaya, M. Spanner, S. De, H. Li, D. Ray, P. Corkum, I. V. Litvinyuk, C. L. Cocke, and M. F. Kling, Phys. Rev. Lett. 112, 113005 (2014); arXiv:1307.0303.

[28] M. Spanner, S. Patchkovskii, E. Frumker, and P. Corkum, Phys. Rev. Lett. 109, 113001 (2012); arXiv:1205.4383.

[29] S. Zhang, C. Lu, T. Jia, Z. Wang, and Z. Sun, Phys. Rev. A 83, 043410 (2011).

[30] M. Machholm and N. E. Henriksen, Phys. Rev. Lett. 87, 193001 (2001)

[31] S. Fleischer, Y. Zhou, R. W. Field, and K. A. Nelson, Phys. Rev. Lett. 107, 163603 (2011); arXiv:1105.1635.

[32] K. Kitano, N. Ishii, and J. Itatani, Phys. Rev. A 84, 053408 (2011).

[33] K. N. Egodapitiya, S. Li, and R. R. Jones, Phys. Rev. Lett. 112, 103002 (2014).

[34] R. Torres, R. de Nalda, and J. P. Marangos, Phys. Rev. A 72, 023420 (2005)

[35] S. Trippel, T. Mullins, N. L. M. Müller, J. S. Kienitz, J. J. Omiste, H. Stapelfeldt, R. González-Férez, and J. Küpper, Phys. Rev. A 89, 051401(R) (2014); arXiv:1401.6897.

[36] J. J. Omiste, M. Gaerttner, P. Schmelcher, R. GonzálezFérez, L. Holmegaard, J. H. Nielsen, H. Stapelfeldt, and J. Küpper, Phys. Chem. Chem. Phys. 13, 18815 (2011).

[37] J. J. Omiste and R. González-Férez, Phys. Rev. A 86, 043437 (2012).

[38] A state $|\tilde{m}, \tilde{n}\rangle$ correlates adiabatically to the field-free state $|m, n\rangle$.

[39] D. Irimia, D. Dobrikov, R. Kortekaas, H. Voet, D. A. van den Ende, W. A. Groen, and M. H. M. Janssen, Rev. Sci. Instrum. 80, 113303 (2009).

[40] F. Filsinger, J. Küpper, G. Meijer, L. Holmegaard, J. H. Nielsen, I. Nevo, J. L. Hansen, and H. Stapelfeldt, J. Chem. Phys. 131, 064309 (2009); arXiv:0903.5413.

[41] J. H. Nielsen, P. Simesen, C. Z. Bisgaard, H. Stapelfeldt, F. Filsinger, B. Friedrich, G. Meijer, and J. Küpper, Phys. Chem. Chem. Phys. 13, 18971 (2011).

[42] S. Trippel, T. G. Mullins, N. L. M. Müller, J. S. Kienitz, K. Długołęcki, and J. Küpper, Mol. Phys. 111, 1738 (2013).

[43] The two-dimensional degree of alignment is defined as $\left\langle\cos ^{2} \theta_{2 \mathrm{D}}\right\rangle=\int_{0}^{\pi} \int_{0}^{r_{\max }} \cos ^{2}\left(\theta_{2 \mathrm{D}}\right) f\left(\theta_{2 \mathrm{D}}, r_{2 D}\right) d r_{2 D} d \theta_{2 \mathrm{D}}$ and the two-dimensional degree of orientation is defined as $\left\langle\cos \theta_{2 \mathrm{D}}\right\rangle=\int_{0}^{\pi} \int_{0}^{r_{\max }} \cos \left(\theta_{2 \mathrm{D}}\right) f\left(\theta_{2 \mathrm{D}}, r_{2 D}\right) d r_{2 D} d \theta_{2 \mathrm{D}}$. $f\left(\theta_{2 \mathrm{D}}, r_{2 D}\right)$ is the projection of the probability density on the 2D screen normalized to 1 .

[44] A detailed discussion on the velocity cut is provided in the Supplemental Material [45].

[45] See Supplemental Material at http://link.aps.org/ supplemental/10.1103/PhysRevLett.114.103003 for a brief discussion on the velocity cut, made on the raw detector images, to determine the degree of orientation.

[46] A. H. Zewail, J. Phys. Chem. A 104, 5660 (2000).

[47] S. L. Liao, T.-S. Ho, H. Rabitz, and S.-I. Chu, Phys. Rev. A 87, 013429 (2013). 